Präv Gesundheitsf 2015 • 10:35-40

DOI 10.1007/s11553-014-0468-8

Online publiziert: 5. September 2014

(c) Die Autor(en) 2014. Dieser Artikel ist auf

Springerlink.com mit Open Access verfügbar
Christiane Wilke · Tobias Elis · Bianca Biallas · Ingo Froböse

Institut für Bewegungstherapie und bewegungsorientierte Prävention und

Rehabilitation an der Deutschen Sporthochschule Köln, Köln, Deutschland

\title{
Gesundheitsbedingte Leistungseinbußen bei der Arbeit durch Präsentismus
}

\section{Einleitung}

Der Einfluss der heutigen Arbeitswelt auf die Gesundheit der Beschäftigten wird zunehmend von den Medien diskutiert. Präsentismus erfährt eine größer werdende Beachtung [17]. Unternehmen erkennen, dass die Gesundheit der Beschäftigten ihre Arbeitsleistung und somit die Wirtschaftlichkeit des Unternehmens beeinflusst. Um gesundheitsförderliche Arbeitsbedingungen zu schaffen, werden zunehmend Maßnahmen des Betrieblichen Gesundheitsmanagements (BGM) durchgeführt. Die vorliegende Studie untersucht die Auswirkungen gesundheitlicher Probleme von Arbeitnehmern auf deren Produktivität.

\section{Problemstellung}

\section{Präsentismusforschung}

Das heute in den Fokus gesetzte Interesse am Phänomen „Präsentismus“ wurde zum ersten Mal in den 1950er Jahren thematisiert. Zunächst stand die Frage im Mittelpunkt, wie Fehlzeiten verringert und die Anwesenheit der Mitarbeiter erhöht werden können - heute vielmehr als Absentismus bekannt. In den 1990er Jahren wurden vermehrt Untersuchungen zu dieser Thematik durchgeführt. In den vornehmlich aus den USA stammenden Studien rückte erstmals der Einfluss von Gesundheitseinbußen auf die Arbeitstätigkeit (Präsentismus) und die Höhe entsprechender Mehrkosten für die Unternehmen in den Blickpunkt [2]. Seitdem steigt das Interesse an der Präsentismusforschung. Es sollen Verluste in der Produktivität durch gezielte Interventionen gemessen und gesenkt werden können. Die Messung von Absentismus anhand von Arbeitsunfähigkeitstagen ist ein gängiges Verfahren. Für den Bereich Präsentismus gibt es ein solches Verfahren bislang nicht, da Präsentismus eine schwer zu erfassende Variable ist.

Während in den USA unter „presenteeism“ die Produktivitätsverluste, die aufgrund von gesundheitlichen Problemen entstehen, und die daraus resultierenden Kosten verstanden werden, umfasst der Begriff „Präsentismus“ in Deutschland zwei verschiedene Punkte (nach [17]):

- Das Verhalten, trotz Krankheit zur Arbeit zu gehen.Einbußen der Produktivität, die dadurch entstehen, dass Mitarbeiter durch gesundheitliche Beschwerden in ihrer Arbeit eingeschränkt sind und unterhalb ihres durchschnittlichen Arbeitspensums bleiben.

Bislang untersuchten nationale Studien Präsentismus primär im Sinne des Verhaltens trotz Krankheit zur Arbeit zu gehen (vgl. [13, 16]). Weitaus weniger Studien haben sich jedoch mit den Produktivitätseinbußen durch Präsentismus befasst. Bödeker u. Hüsing [1] untersuchten 2007 rund 2000 Erwerbstätige in Deutschland zum Einfluss von Krankheit auf die Produktivität. Die Ergebnisse ergeben, dass sich rund $59 \%$ der Befragten in ihrer Produktivität eingeschränkt fühlen. Dies entspreche einem Produktivitätsverlust durch Krankheit von $20 \%$. Iverson $u$. Krause [8] haben zudem ein Berechnungsmodell für Produktivitätsverluste durch Krankheit erstellt. Grundlage des Berechnungsmodells sind internationale Forschungsstudien und bundesdeut- sche Prävalenzdaten. Das Modell zielt auf eine vorsichtige Schätzung der Produktivitätseinbußen durch Präsentismus und Absentismus in deutschen Unternehmen $\mathrm{ab}$ und versucht die häufigsten sowie teuersten Krankheiten als Gründe zu identifizieren [3, 4]. Die wesentlichen Aussagen des Modells sind:

- Es existieren 13 Gesundheitsprobleme, die Produktivitätsverluste bei der Arbeit verursachen. Kopfschmerzen, Schlafstörungen, Allergien, Depressionen, Stress und Rückenschmerzen treten am häufigsten auf

- 10-15\% der produktiven Zeit geht aufgrund von Präsentismus und Absentismus verloren. Dabei entfallen ein Drittel auf Absentismus und zwei Drittel auf Präsentismus.

- Im Durchschnitt entspricht dies einem Verlust von jährlich 27 Tagen pro Mitarbeiter.

Die vorgestellten Studien zeigen, dass die Produktivitätsverluste aufgrund von Leistungseinbußen sehr unterschiedlich sind. Nach einer Analyse aller vorliegenden Studien beziffern Fissler u. Krause [3] die Höhe der Produktivitätsverluste auf $3,031,7 \%$. Bislang liegen noch keine Studienergebnisse zu möglichen Beeinträchtigungen, deren Ursachen und Wirkung auf die Produktivität vor. Dies gründet sich vermutlich darin, dass sich die Forschung bislang mit dem Vorliegen und den Folgen chronischer Erkrankungen für die Produktivität, nicht aber mit möglichen Ursachen von Präsentismus befasst hat [17].

Die europäische Präsentismusforschung konzentriert sich in der Analyse möglicher Gründe insbesondere auf die 


\begin{tabular}{|lll|}
\hline \multicolumn{3}{|l}{ Tab. 1 Stichprobenbeschreibung } \\
\hline \multicolumn{3}{|c|}{$\mathbf{n}$} \\
\hline Geschlecht & & \\
\hline Männlich & 211 & 80,5 \\
\hline Weiblich & 51 & 19,5 \\
\hline Gesamt & 262 & 100,0 \\
\hline Alter & & \\
\hline$<40$ Jahre & 119 & 45,4 \\
\hline$>$ 40 Jahre & 143 & 54,6 \\
\hline Gesamt & 263 & 100,0 \\
\hline Tätigkeitsbereich & & \\
\hline Kaufmännisch & 113 & 43,1 \\
\hline Gewerblich & 149 & 56,9 \\
\hline Gesamt & 262 & 100,0 \\
\hline
\end{tabular}

Leistungsfähigkeit und die gesundheitlichen (Langzeit-)Folgen für den Arbeitnehmer. Trotz der Anwesenheit des Arbeitnehmers kann die Leistungsfähigkeit durch gesundheitliche Einbußen gemindert sein. Denn obwohl Arbeitnehmer physisch am Arbeitsplatz präsent sind, können sie keine Spitzenleistungen erbringen. Zudem steigt bei reduzierter Aufmerksamkeit die Fehlerwahrscheinlichkeit [14].

In einer dänischen Studie konnte gezeigt werden, dass eine gemiedene Krankmeldung zu einer Verschleppung der Krankheit und damit zu einem potentiell längeren krankheitsbedingten Ausfall führen kann. In der Studie weisen Beschäftigte, die mehr als 6-mal im Jahr krank zur Arbeit gingen, ein um 74\% höheres Risiko auf, zu einem späteren Zeitpunkt länger als 2 Monate krankheitsbedingt auszufallen als andere Beschäftigte [7]. Neben den persönlichen Folgen für den Arbeitnehmer kann die Anwesenheit trotz Krankheit negative Folgen für Kollegen durch Ansteckung bei etwa infektiösen Krankheiten haben [17].

Die vorliegende Arbeit untersucht die Ermittlung gesundheitsbedingter Leistungseinbußen während der Arbeit in einem mittelständischen Unternehmen, die infolge von Präsentismus entstehen. Es wird zudem analysiert, inwieweit sich die Einschränkungen in den Anforderungsbereichen hinsichtlich Geschlecht, Alter und Arbeitssektor differenzieren. Daraus leiten sich folgende Fragestellungen ab:

- Treten in einem mittelständischen

Unternehmen gesundheitsbeding- te Leistungseinschränkungen bei der Arbeit auf?

- Gibt es Unterschiede zwischen gewerblichen (Produktion) und kaufmännischen Mitarbeitern bezüglich der Höhe der Leistungseinbußen sowie der Entstehung von Präsentismus unter Berücksichtigung der abgefragten Anforderungsbereiche?

- Gibt es Unterschiede zwischen den Geschlechtern und der Höhe der Leistungseinbußen sowie der Entstehung von Präsentismus unter Berücksichtigung der abgefragten Anforderungsbereiche?

- Gibt es Unterschiede zwischen beim Alter der Beschäftigten und der Höhe der Leistungseinbußen sowie der Entstehung von Präsentismus unter Berücksichtigung der abgefragten Anforderungsbereiche?

\section{Methodik}

\section{Stichprobe}

Die Untersuchung findet in einem mittelständischen Unternehmen in der Chemiebranche statt. Für die Untersuchung kommen 468 von insgesamt 627 Personen in Betracht. Ausgenommen sind Angestellte des Außendienstes, Auszubildende, Leasingkräfte und Personal in Elternzeit bzw. Mutterschutz. 277 Mitarbeiter/-innen nehmen schließlich an der Befragung teil, was eine Rücklaufquote von $59,1 \%$ bedeutet. Das Durchschnittsalter liegt bei 41,2 Jahren, wobei über die Hälfte $(54,6 \%)$ der Angestellten über 40 Jahre alt sind. Die überwiegende Belegschaft ist männlich $(80,5 \%)$ und dem gewerblichen Bereich im Unternehmen zu zuteilen $(56,9 \%$, • Tab. 1$)$.

Entsprechend der unterschiedlichen Tätigkeit in Produktion und Verwaltung variieren die physischen und psychischen Belastungskriterien. Der kaufmännische Bereich, der überwiegend aus Bildschirmarbeitsplätzen mit sitzender Tätigkeit besteht, stellt insbesondere psychische Belastungen (u. a. Stress, Leistungsdruck) dar. Der gewerbliche Bereich hingegen beschreibt v. a. körperliche Tätigkeiten und umfasst daher primär physische Belastungen.

\section{Messinstrumente und Untersuchungsdurchführung}

Die gesundheitsbedingten Leistungseinbußen bei der Arbeit sowie bestehende Schwierigkeiten in der Ausführung spezieller Arbeitsanforderungen werden mit Hilfe des Fragebogens ,Work Limitations Questionnaire“(WLQ) erhoben [12]. Der WLQ misst das Ausmaß der Auswirkung gesundheitlicher Probleme der Beschäftigten auf das Vermögen, bestimmte Tätigkeiten innerhalb ihrer Berufstätigkeit zu erledigen. Er umfasst 25 Items zu speziellen Anforderungen während der Arbeit. Die Beantwortung erfolgt in Form einer 5-Punkte-Likert-Skala. Die Werte der Items rangieren auf einer Skala von $0(\mathrm{zu}$ keiner Zeit eingeschränkt) und 100 (ständig eingeschränkt).

Die Items lassen sich vier Tätigkeitsbereichen zuordnen: Zeitmanagement, körperliche, mental-interpersonelle und Leistungsanforderungen [11]. Die Fragen richten sich an sowohl körperliche als auch seelische Probleme, die sich auf akute und chronische Erkrankungen sowie entsprechende Therapien beziehen. Das Aufsummieren der vier Dimensionen lässt eine Aussage über die verlorene Produktivität (Produktivitätsverlust) im Ganzen zu. Die Validität $(>0,70)$ und Reliabilität $(>0,90)$ wurden in verschiedenen Studien nachgewiesen $[12,18,20]$.

Zusätzlich wird dem Fragebogen eine weitere Seite zur Erfassung von Geschlecht, Alter und Arbeitsbereich beigefügt, um eine differenzierte Auswertung zu ermöglichen. Bei der Auswertung handelt es sich um eine Schätzung der prozentualen Unterschiede in der Arbeitsleistung im Vergleich zu den Beschäftigten, die keine Arbeitseinschränkungen aufgrund gesundheitlicher Probleme aufweisen [11]. Die Angestellten des Unternehmens werden vorab via Intranet, E-Mail, Aushänge oder persönliche Ansprachen über die Befragung informiert. Dazu gehören die Vorstellung der Thematik, des Fragebogens, der Durchführung und des Datenschutzes. Aufgrund des Drei-Schicht-Systems im Unternehmen ist die Kommunikation in der Produktion erschwert, da die Mitarbeiter keinen Zugang zu einem PC (E-Mail) besitzen. Um die Motivation der Beschäftigten an der Befragung teil- 
zunehmen dennoch zu erhöhen und Fragen beantworten zu können, wurde hier das Hauptaugenmerk auf die Kommunikation in persönlichen Ansprachen gelegt.

\section{Statistisches Verfahren}

Die Daten des Fragebogens werden zur Verwaltung und weiteren Bearbeitung im Datenverarbeitungsprogramm Excel $\left(\right.$ Microsoft $\left.^{\odot}\right)$ eingegeben. Zur weiteren statistischen Auswertung der Daten wird das Programm SPSS $\left(\mathrm{IBM}^{\odot}\right)$ genutzt. Die Prüfung auf mögliche Mittelwertunterschiede erfolgt mittels t-Test für unabhängige Stichproben.

\section{Ergebnisse}

Von den 277 Fragebögen (Rücklaufquote: 59,6\%) konnten letztlich 262 zur Auswertung herangezogen werden (Auswertquote: $56,0 \%)$.

Die gesundheitsbedingten Produktivitätsverluste im Unternehmen in den vergangenen 14 Tagen werden durch den WLQ auf insgesamt $4,1 \%$ geschätzt. Die Produktivitätsverluste der Männer $(4,3 \%)$ fallen signifikant höher aus als die der Frauen $(3,1 \% ; p<0,01)$. Der Vergleich der beiden Arbeitsbereiche zeigt, dass die gesundheitsbedingten Leistungseinbußen in der Produktion (4,7\%) signifikant größer sind als im kaufmännischen Bereich (3,2\%; p<0,001). Die Ergebnisse beider Altersklassen $(<40$ Jahre, $>40$ Jahre) im Vergleich verdeutlichen, dass die über 40-Jährigen (4,3\%) höhere Produktivitätsverluste aufweisen als die unter 40-Jährigen (3,7\%). Dieser Unterschied ist jedoch nicht signifikant ( $>$ > 0,05; • Tab. 2).

Die Analyse der gesundheitsbedingten Leistungseinschränkungen in den vier Anforderungsbereichen gibt Auskunft darüber, wie häufig es Schwierigkeiten gibt, die speziellen Anforderungen auszuführen. Es zeigt sich, dass sich Männer und Frauen lediglich in den Bereichen „Zeitmanagement" und „körperliche Anforderungen“ signifikant voneinander unterscheiden ( $\mathrm{p}<0,01 ; \mathrm{p}<0,001)$. Die Dimensionen „mental-interpersonelle Anforderungen“ und „Leistungsanforderungen" weisen keine signifikanten Ergebnisse auf ( $p>0,05)$. Hinsichtlich des

Präv Gesundheitsf 2015 • 10:35-40 DOI 10.1007/s11553-014-0468-8

(c) Die Autor(en) 2014. Dieser Artikel ist auf Springerlink.com mit Open Access verfügbar

\section{Wilke $\cdot$ T. Elis · B. Biallas · I. Froböse}

\section{Gesundheitsbedingte Leistungseinbußen bei der Arbeit durch Präsentismus}

\section{Zusammenfassung}

Hintergrund. Das Verhalten, trotz Krankheit zur Arbeit zu gehen erzeugt für Arbeitgeber hohe Kosten und beeinträchtigt die Gesundheit der Mitarbeiter. Maßnahmen des Betrieblichen Gesundheitsmanagements (BGM) erhalten und fördern diesbezüglich die Gesundheit der Beschäftigten.

Methode. An der Befragung nahmen 277 Mitarbeiter/-innen aus Produktion (56,9\%) und Verwaltung $(43,1 \%)$ teil. Das Durchschnittsalter betrug 41,2 Jahre, der männliche Anteil 80,5\%. Erfasst wurden Produktivitätsverluste und bestehende Schwierigkeiten spezieller Arbeitsanforderungen (Zeitmanagement, Leistungs-, körperliche sowie mental-interpersonelle Anforderungen). Ergebnisse. Die Produktivitätsverluste durch Präsentismus sind gering (4,1\%). Männer und Mitarbeiter der Produktion erzeugen höhere Produktionsverluste $(p<0,01 ; p<0,001)$ und weisen mehr Probleme hinsichtlich Zeitmanagement $(p<0,01)$ und körperliche Anforderungen $(p<0,001)$ auf. Ältere haben mehr Probleme im Bereich der körperlichen Anforderungen $(p<0,001)$.

Schlussfolgerung. Präsentismus wird zukünftig zentral für die "Gesundheit am Arbeitsplatz" sein. Das BGM kann durch unterschiedliche Messinstrumente Ursachen und Folgen von Präsentismus erfassen.

\section{Schlüsselwörter}

Gesundheitsmanagement, betriebliches . Produktivitätsverluste · Arbeitsanforderungen . Gesundheit am Arbeitsplatz .

Zeitmanagement

\section{Health-related performance loss during work caused by presenteeism}

\section{Abstract}

Background. Going to work sick poses high costs to the employer and affects the employee's health. Occupational health management measures enhance the employee's health and counter the presenteeism debate. Methods. A total of 277 employees from two working areas (manufacturing: 56.9\%; administration: $43.1 \%$ ) participated in a survey. The average age was 41.2 years, $80.5 \%$ were male. The loss of productivity as well as existing troubles with specific job requirements (time management, physical and mental as well as performance requirements) were evaluated.

Results. The company's loss of productivity is estimated as low (4.1\%). A higher loss of productivity is associated with men $(<0.01)$ and manufacturing employees $(<0.001)$. These two groups also have more troubles with respect to time management $(<0.01)$ and physical demands $(<0.001)$. Regarding age, older employees had more troubles only with physical demands $(<0.001)$.

Conclusion. Presenteeism is said to be vital in terms of occupational health in the next few years. Occupational health management uses different tools and, thus, helps to identify the cause and consequence of presenteeism.

\section{Keywords}

Occupational health management . Productivity loss - Job requirement . Employee health - Time management
Tätigkeitsbereichs werden ähnliche Ergebnisse deutlich. Kaufmännische und gewerbliche Angestellte unterscheiden sich signifikant in den Bereichen „Zeitmanagement“ $(p<0,01)$ und „körperlichen Anforderungen“ $(p<0,001)$. Die Dimensionen „mental-interpersonelle Anforderungen“ und „Leistungsanforderungen“ bleiben nicht signifikant ( $\mathrm{p}>0,05)$. Im Vergleich der beiden Altersgruppen unterscheiden sich Beschäftigte unter 40 Jahren und über 40-Jährige nur im Be- reich der körperlichen Anforderungen signifikant voneinander $(\mathrm{p}<0,001)$. Die Dimensionen „körperlichen Anforderungen“ „mental-interpersonelle Anforderungen" und „Leistungsanforderungen“" sind nicht signifikant ( $p>0,05$; $~ T a b .3)$. 
Tab. 2 Relative Produktivitätsverluste im

Vergleich der Faktoren Geschlecht, Tätig-

keitsbereich und Alter mit dem Mittelwert

$(\mathrm{m})$, der Standardabweichung (s) und der

Signifikanz (p)

\begin{tabular}{|c|c|c|c|}
\hline & $\mathrm{n}$ & $\begin{array}{l}\text { Produktions- } \\
\text { verlust }(m \pm s)\end{array}$ & $p$ \\
\hline \multicolumn{4}{|c|}{ Geschlecht } \\
\hline Männer & 211 & $4,3 \pm 3,6$ & \multirow[t]{2}{*}{$<0,01$} \\
\hline Frauen & 51 & $3,1 \pm 3,7$ & \\
\hline Gesamt & 262 & $4,1 \pm 3,6$ & \\
\hline \multicolumn{4}{|c|}{ Tätigkeitsbereich } \\
\hline $\begin{array}{l}\text { Kauf- } \\
\text { männisch }\end{array}$ & 113 & $3,2 \pm 3,2$ & \multirow[t]{2}{*}{$<0,001$} \\
\hline $\begin{array}{l}\text { Gewerb- } \\
\text { lich }\end{array}$ & 149 & $4,7 \pm 3,8$ & \\
\hline Gesamt & 262 & $4,1 \pm 3,6$ & \\
\hline \multicolumn{4}{|l|}{ Alter } \\
\hline $\begin{array}{l}<40 \\
\text { Jahre }\end{array}$ & 119 & $3,7 \pm 3,1$ & \multirow[t]{2}{*}{$\geq 0,05$} \\
\hline $\begin{array}{l}>40 \\
\text { Jahre }\end{array}$ & 143 & $4,3 \pm 4,0$ & \\
\hline Gesamt & 262 & $4,3 \pm 3,6$ & \\
\hline
\end{tabular}

\section{Diskussion}

\section{Methode}

Die Auswertquote in der vorliegenden Studie beträgt 56,0\% und ist im Hinblick auf die Befragungsinhalte und mögliche Einflussfaktoren (Betriebsklima, Arbeitsbedingungen und -umfeld, Datenschutz) als hoch zu werten.

Die Erhebung von Informationen über die Beschäftigten eines Unternehmens kann über verschiedene Verfahren erfolgen: Simulationen standardisierter Tätigkeiten unter Testbedingungen, eine Messung objektiver Daten sowie eine subjektive Selbsteinschätzung der Beschäftigten. Die Nutzung von Selbsteinschätzungsinstrumenten wird erst seit etwa einer Dekade systematisch zur Bestimmung von Gesundheit und Produktivität am Arbeitsplatz eingesetzt [4]. Die Vorteile liegen v. a. in einem zeitlich, personell und finanziell geringem Aufwand und der daraus resultierenden Erleichterung für die Erhebung der Daten. Aus diesem Grund wurde die Befragung mittels WLQ gewählt. Der WLQ erfüllt darüber hinaus die Gütekriterien Validität, Reliabilität und Objektivität in ausreichendem Maße $[17,18]$.

Tab. 3 Anteile der Arbeitszeit, in denen Schwierigkeiten der Beschäftigten auftreten, den Anforderungsbereichen gerecht zu werden, unterteilt nach Geschlecht, Tätigkeitsbereich und Alter

\begin{tabular}{|c|c|c|c|c|c|}
\hline & $\mathrm{n}$ & $\begin{array}{l}\text { Zeitmanage- } \\
\text { ment }\end{array}$ & $\begin{array}{l}\text { Körperliche } \\
\text { Anforderungen }\end{array}$ & $\begin{array}{l}\text { Mental-inter- } \\
\text { personelle An- } \\
\text { forderungen }\end{array}$ & $\begin{array}{l}\text { Leistungsanfor- } \\
\text { derungen }\end{array}$ \\
\hline \multicolumn{6}{|l|}{ Geschlecht } \\
\hline Männer & 211 & 16,8 & 24,3 & 13,6 & 14,0 \\
\hline Frauen & 51 & 11,0 & 9,8 & 11,1 & 11,7 \\
\hline$p$ & & $<0,01$ & $<0,001$ & $>0,05$ & $>0,05$ \\
\hline \multicolumn{6}{|c|}{ Tätigkeitsbereich } \\
\hline $\begin{array}{l}\text { Kaufmän- } \\
\text { nisch }\end{array}$ & 113 & 12,1 & 10,1 & 11,4 & 12,0 \\
\hline $\begin{array}{l}\text { gewerb- } \\
\text { lich }\end{array}$ & 149 & 18,4 & 30,0 & 14,5 & 14,6 \\
\hline$p$ & & $<0,01$ & $<0,001$ & $>0,05$ & $>0,05$ \\
\hline \multicolumn{6}{|l|}{ Alter } \\
\hline$<40$ Jahre & 119 & 14,3 & 17,0 & 12,9 & 12,4 \\
\hline$>40$ Jahre & 143 & 16,8 & 25,1 & 13,4 & 14,4 \\
\hline $\mathrm{p}$ & & $>0,05$ & $<0,001$ & $>0,05$ & $>0,05$ \\
\hline \multicolumn{6}{|l|}{ Gesamt } \\
\hline Gesamt & 262 & 15,6 & 21,5 & 13,1 & 13,5 \\
\hline
\end{tabular}

Aufgrund der Tatsache, dass lediglich bei 3,9\% der beantworteten Fragebögen keine Werte für die verlorene Produktivität errechnet werden konnten, ist in dieser Studie von einer hohen Praktikabilität des WLQ auszugehen. Zudem nimmt der WLQ mit nur 25 Fragen einen zeitlich geringen Umfang ein. Im Hinblick auf die Gesamtthematik des Präsentismus erhebt der WLQ allerdings keine Daten über mögliche Ursachen, Einflussfaktoren und Folgen von Präsentismus. Somit lassen sich die Ergebnisse des WLQ nicht direkt in monetäre Größen umrechnen oder sich unmittelbare Handlungsfelder des BGM ableiten.

\section{Ergebnisse}

Die vorliegende Untersuchung eines mittelständischen Chemieunternehmens gibt einen Hinweis darauf, dass gesundheitsbedingte Produktivitätsverluste bei der Arbeit auftreten. Die Produktivitätsverluste belaufen sich auf $4,1 \%$, fallen im Vergleich zu anderen deutschen Studien mit Verlusten von $11 \%$ und mehr aber eher gering aus $[1,20]$. Im Vergleich zu einer US-amerikanischen Studie (2,8\%) fällt der Produktivitätsverlust im untersuchten Unternehmen höher aus [10]. Weitere Studien kommen jedoch zu dem Er- gebnis, dass die Verlustquote in anderen Unternehmen wesentlich höher ist $[6,15]$.

Gründe für eine teilweise geringere Verlustquote in den USA liegen v. a. darin, dass bereits seit den 1950er Jahren eine aktive Auseinandersetzung mit der Thematik Anwesenheit am Arbeitsplatz stattfindet und sich daraus die Präsentismusforschung ableitete $[17,19]$. Als Vorreiter ist die National Aeronautics and Space Administration (NASA) zu nennen, die sich bereits Anfang der 1970er Jahre mit der Thematik beschäftigte und zeitgleich erste präventive Gesundheitsprogramme durchführte [10].

In der Literatur werden unterschiedliche Einflussfaktoren auf den Präsentismus genannt. Insbesondere die Faktoren Alter, Geschlecht und Beruf werden oft diskutiert und sind in diesem Zusammenhang von hoher Bedeutung. In Bezug auf den Tätigkeitsbereich innerhalb des Unternehmens zeigen die Ergebnisse, dass gewerbliche Mitarbeiter einen höheren Produktivitätsverlust durch Präsentismus aufweisen als kaufmännisch Angestellte, was durch einen signifikanten Unterschied bekräftigt wird. Ergebnisse anderer Studien werfen auf, dass der kaufmännische Bereich höhere Produktionsverluste aufweist als der gewerbliche Bereich [20]. Gründe für die Unterschiede 
der vorliegenden Studie liegen hier insbesondere in den körperlichen Belastungen, die die Produktionsmitarbeiter häufiger zu einem Fernbleiben vom Arbeitsplatz zwingen. Mitarbeitern des kaufmännischen Bereichs ist es hingegen besser möglich trotz gesundheitlicher Probleme ihrer Arbeit nachzugehen. In Bezug auf die Studie von Wallat [20] liegt nahe, dass Unterschiede in den beruflichen Tätigkeiten zu verschiedenen Ergebnissen als in der vorliegenden Studie führen. In Zukunft muss gewährleistet sein, dass Studien im Sinne der Vergleichbarkeit den gleichen Sachverhalt untersuchen.

Die Ergebnisse der Untersuchung geben weiterhin einen Hinweis darauf, dass Männer einen höheren Produktivitätsverlust durch Präsentismus aufweisen als Frauen. Insbesondere die amerikanische Betrachtungsweise des Präsentismus fokussiert die Prävalenz chronischer Erkrankungen und die daraus resultierenden Produktivitätsverluste [15]. Sollte also ein Zusammenhang zwischen chronischen Erkrankungen und der Höhe der Produktivitätsverluste bestehen, müssten Prävalenzdaten zu chronischen Erkrankungen diese Hinweise der vorliegenden Studie bestätigen. Entsprechende Daten aus dem Jahr 2009 zeigen jedoch, dass Frauen $(42,3 \%)$ offter angeben an einer chronischen Erkrankung zu leiden als Männer (35,8\%). Der Hinweis, dass Männer höhere Produktivitätsverluste als Frauen aufzeigen, kann daher nicht weiter untermauert werden.

Hinsichtlich des Alters wird auf den ersten Blick deutlich, dass jüngere Mitarbeiter $(<40$ Jahre) einen geringeren Produktivitätsverlust haben als ältere Mitarbeiter (<40 Jahre; • Tab. 3). Die statistische Auswertung konnte jedoch keinen signifikanten Unterschied feststellen. Daher kann in dieser Untersuchung nicht belegt werden, dass sich die gesundheitsbedingten Produktivitätsverluste von jüngeren und älteren Mitarbeitern unterscheiden. Der Zusammenhang zwischen den Faktoren Alter und Gesundheit wird nach wie vor kontrovers diskutiert, da es sowohl empirische Hinweise auf eine Verbesserung des Gesundheitszustands älterer Menschen gibt als auch auf eine Ausweitung der Morbidität [21].

\section{Fazit für die Praxis}

Im Rahmen des demographischen Wandels wird vermutet, dass Präsentismus zukünftig einer der zentralen Schwerpunkte sein wird, um eine nachhaltige Wettbewerbsfähigkeit der Unternehmen zu sichern [17]. Es mangelt bislang an Vergleichsstudien, insbesondere in Deutschland herrscht noch keine Einheit über mögliche Ursachen und Folgen von Präsentismus. Bislang hat sich die Forschung mit der Wechselseitigkeit zwischen chronischen Erkrankungen und der Produktivität, nicht aber mit den Ursachen von Präsentismus befasst. In Zukunft wäre es wünschenswert, dass Unternehmen den Einsatz von unterschiedlichen Messinstrumenten unterstützen, um die Mehrdimensionalität von Präsentismus zu erfassen. Neben der Erhebung gesundheitsbedingter Produktivitätsverluste ermöglichen Maßnahmen des BGM die Identifizierung möglicher Ursachen von Präsentismus durch spezifische Analysen der Arbeitsunfähigkeitsdaten, präventiv-medizinische Untersuchungen und Gesundheitsangebote.

\begin{tabular}{ll}
\hline Korrespondenzadresse \\
\hline \\
$\begin{array}{l}\text { Institut für } \\
\text { Bewegungstherapie und } \\
\text { bewegungsorientierte } \\
\text { Prävention und Rehabilitation } \\
\text { an der Deutschen } \\
\text { Sporthochschule Köln } \\
\text { Am Sportpark Müngersdorf 6 } \\
\text { 50933 Köln } \\
\text { wilke@dshs-koeln.de }\end{array}$ \\
\hline
\end{tabular}

\section{Einhaltung ethischer Richtlinien}

Interessenkonflikt. C. Wilke, T. Elis, B. Biallas und I. Froböse geben an, dass kein Interessenkonflikt vorliegt.

Dieser Beitrag beinhaltet keine Studien an Menschen oder Tieren.

Open Access. Dieser Artikel unterliegt den Bedingungen der Creative Commons Attribution License. Dadurch sind die Nutzung, Verteilung und Reproduktion erlaubt, sofern der/die Originalautor/en und die Quelle angegeben sind.

\section{Literatur}

1. Bödeker W, Hüsing T (2007) IGA-Report 12- IGABarometer 2. Welle. Einschätzungen der Erwerbsbevölkerung zum Stellenwert der Arbeit, zur Verbreiterung der Akzeptanz von betrieblicher Prävention und zur krankheitsbedingten Beeinträchtigung der Arbeit. Essen

2. Burton W, Conti D (1999) The real measure of productivity. Bus Health 17(11) 34-36

3. Fissler ER, Krause R (2010) Absentismus, Präsentismus und Prosuktivität. In: Badura B, Walter U, Hehlmann T (Hrsg) Betriebliche Gesundheitspolitik. Der Weg zur gesunden Organisation. Berlin Heidelberg, Springer. 411-425

4. Fissler E (2011) Präentismus-Mangement in der Praxis: Produktivität messen, analysieren und verbessern. In: Corporate Health Jahrbuch. Betriebliches Gesundheitsmanagement in Deutschland. Hoehner Research \& Consulting Group, Bonn

5. Fuchs T, Kistler E, Trischler F (2009) DGB-Index Gute Arbeit. Exemplarische Ergebnisse und Diskussion. In: Kistler E, Mußmann F (Hrsg) Arbeitsgestaltung als Zukunftsaufgabe. Hamburg

6. Goetzel R, Long S, Ozminkowski R, Hawkins K, Wang S, Lynch W (2004) Health, absence, disability, and presenteeism cost estimates of certain physical and mental health conditions affecting U.S. employers. J Occ Environ Med 46(4):398-412

7. Hansen C, Andersen J (2009) Sick at work-a risk factor for long-term sickness absence at a later date? J Epidemiol Commun H 63(5):397-402

8. Iverson DC, Krause R (2007) Produktivitätsräuber Präsentismus. Personal H. 12:46-48

9. Hemp P (2005) Krank am Arbeitsplatz. Harvard Bus Manag 1:47-60

10. Lerner D, Guindon A (2008) HRA/WLQ Results \& Analysis for NASA. http://ohp.nasa.gov. Zugegriffen: 13. März 2012

11. Lerner D, Henke R (2008) What does research tell us about depression, job performance, and work productivity? J Occ Environ Med 50(4):401-410

12. Lerner D, Amick B, Rogers W, Malspeis S, Bungay $K$, Cynn D (2001) The work limitations questionnaire. Med Care 39(1):72-85

13. Lindner M, Sutz R, Woldt C (2009) DGB - Index Gute Arbeit. Der Report 2009. Wie die Beschäftigten die Arbeitswelt in Deutschland beurteilen. DGB, Berlin

14. Middaugh D (2007) Presenteeism: sick and tired at work. Dermatol Nurs 19(2):172-173, 185

15. Riedel J, Grossmeier J, Haglund-Howieson L, Buraglio C, Anderson D, Terry P (2009) Use of a normal impairment factor in quantifying avoidable productivity loss because of poor health. J Occ Environ Med 51(3) 283-295

16. Schmidt J, Schröder H (2010) Präsentismus - Krank zur Arbeit aus Angst vor Arbeitsplatzverlust. In: Badura B, Schröder H, Klose J, Macco K (Hrsg) Fehlzeiten-Report. Arbeit und Psyche: Belastungen reduzieren - Wohlbefinden fördern. Springer, Heidelberg Berlin New York

17. Steinke M, Badura B (2011) Präsentismus: Ein Review zum Stand der Forschung. Baua, Dortmund

18. Tang K, Beaton D, Boonen A, Gignac M, Bombardier C (2011) Measures of work disability and productivity: Rheumatoid Arthritis Specific Work Productivity Survey (WPS-RA), Workplace Activity Limitations Scale (WALS), Work Instability Scale for Rheumatoid Arthritis (RA-WIS), Work Limitations Questionnaire (WLQ), and Work Productivity and Activity Impairment Questionnaire (WPAI). Arthritis Care Res 63(11):337-349 


\section{Arbeitswelt}

19. Uris A (1955) How to build Presenteeism. Petroleum Refiner 34:348-359

20. Wallat $F$ (2007) Gesundheit und Produktivität im Unternehmen - Eine empirische Analyse am Beispiel der Henkel KGaA. Krefeld Hochschule Niederrhein, Krefeld

21. Winter M, Maaz A, Kuhlmey, A (2006) Ambulante und stationäre medizinische Versorgung im Alter. Bundesgesundheitsblatt - Gesundheitsforschung - Gesundheitsschutz 49(6):575-582 